\title{
UPDATED ELECTRON-CLOUD SIMULATION RESULTS FOR THE LARGE HADRON COLLIDER LHC*
}

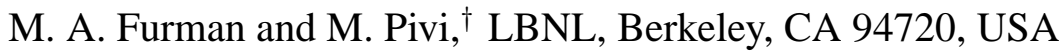

\begin{abstract}
This paper presents new simulation results for the power deposition from the electron cloud in the beam screen of the Large Hadron Collider (LHC). We pay particular attention to the sensitivity of the results to certain low-energy parameters of the secondary electron (SE) emission. Most of these parameters, which constitute an input to the simulation program, are extracted from recent measurements at CERN and SLAC.
\end{abstract}

\section{INTRODUCTION.}

In the LHC, the synchrotron radiation will lead to a large number of photoelectrons from the beam screen wall. These photoelectrons will be accelerated by the electric field of the beam, and may create secondary electrons upon hitting the chamber walls, which may be accelerated in turn by successive bunches. Under conditions where the motion of the secondary electrons are generated in phase with the bunch passages, and if the secondary electron yield (SEY) on the surface on average exceeds unity, the resultant electron cloud may increase significantly during few bunch passages, and may ultimately cause undesired effects such as a large heat load on the wall, vacuum pressure increase, and e-p coupling leading, possibly, to beam instability. Since the total heat load budget for the cryogenic system is $1 \mathrm{~W} / \mathrm{m}$, the power deposited on the beam screen by the electrons is a main concern for the LHC. The electron cloud in the LHC has been extensively studied for the last four years $[1,2]$. In this paper we present updated simulation results obtained with the electron-cloud effect (ECE) code that has been developed at LBNL over the past five years [3]. We are particularly interested in reproducing and understanding results obtained at CERN [4] within the context of our model for the SEY.

When an electron hits the wall, it generates secondary electrons with an energy distribution that is not well determined experimentally. The details of this emitted-electron energy distribution are important for the development of the electron cloud. In this paper, we investigate the sensitivity of the power deposited on the beam screen to several parameters of the model for the SE energy distribution. We follow as much as possible a fit to experimental data on the SEY obtained from recent bench measurements at SLAC. Furthermore, we discuss the effects of the SEY at very low incident electron energy, a parameter that is difficult to measure, and that is currently under study.

\footnotetext{
* Work supported by the SNS project and by the US DOE under contract DE-AC03-76SF00098.

†mafurman@lbl.gov and mpivi@lbl.gov
}

\section{PHYSICAL MODEL}

\subsection{Sources of electrons}

In this article we consider the dominant sources of electrons in the LHC, namely: photoelectrons arising from the synchrotron radiation hitting the walls of the vacuum chamber, and secondary emission from electrons hitting the walls. Although our code accommodates other sources of electrons, such as residual gas ionization, we have turned them off for the purposes of this article.

Table 1: Simulation parameters for the LHC.

\begin{tabular}{l|l|r}
\hline \hline Parameter & Symbol & Value \\
\hline Proton beam energy & $E$ & $7 \mathrm{TeV}$ \\
Dipole field & $B$ & $8.4 \mathrm{~T}$ \\
Bunch population & $N_{p}$ & $1.05 \times 10^{11}$ \\
Bunch spacing & $\tau_{g}$ & $25 \mathrm{~ns}$ \\
Bunch length rms & $\sigma_{z}$ & $7.7 \mathrm{~cm}$ \\
Trans. bunch size & $\sigma_{x}=\sigma_{y}$ & $300 \mu \mathrm{m}$ \\
Beam pipe semi-axes & $a, b$ & $22,18 \mathrm{~mm}$ \\
No. kicks/bunch & $N_{k}$ & 51 \\
No. steps bet. buckets & $N_{g}$ & 20 \\
Photon reflectivity & $R$ & $10 \%$ \\
SEY params. initial & $\delta_{\max }, E_{\max }$ & $1.9,240 \mathrm{eV}$ \\
SEY params. final & $\delta_{\max }, E_{\max }$ & $1.1,170 \mathrm{eV}$ \\
Quantum efficiency & $Y_{i}^{\prime} \div Y_{f}^{\prime}$ & $0.05 \div 0.025$ \\
\hline \hline
\end{tabular}

We represent the SEY $\delta\left(E_{0}\right)$ by a close fit to the Seiler parametrization [5], given explicitly in Ref. 3. The main SEY parameters are the energy $E_{\max }$ at which $\delta\left(E_{0}\right)$ is maximum $\left(E_{0}=\right.$ incident electron energy), and the peak value itself, $\delta_{\max }=\delta\left(E_{\max }\right)$, see Table 1 . For the purpose of these simulations, we represent the corresponding emitted-electron energy spectrum $d \delta / d E(E=$ emitted secondary energy) by two different models: (1) a halfGaussian secondary spectrum, and (2) a full fit to the data. The half-Gaussian model is implemented in our code by adjusting the single-electron energy distribution functions in such a way that $d \delta / d E$ is a Gaussian in $E$ centered at $E=0$ with $5 \mathrm{eV}$ rms spread and restricted, of course, to $E \geq 0$. The full-fit model is described elsewhere $[3,6]$. It is implemented by adjusting parameters such that $d \delta / d E$ and $\delta\left(E_{0}\right)$ fit the measured data of copper [7]. A sample is shown in Fig. 1 for $E_{0}=300 \mathrm{eV}$.

As a variant to the full-fit model, we have also switched off the contributions of the rediffused and the elastically reflected electrons, thereby keeping only the true-secondary 
component. The value of the SEY at incident electron energies $\lesssim 10 \mathrm{eV}$ is an important parameter since it determines the accumulation rate and the electron cloud intensity itself. With this motivation we have repeated our simulations under the assumption $\delta(0)=0.6$, as an additional variant of the full-fit model.

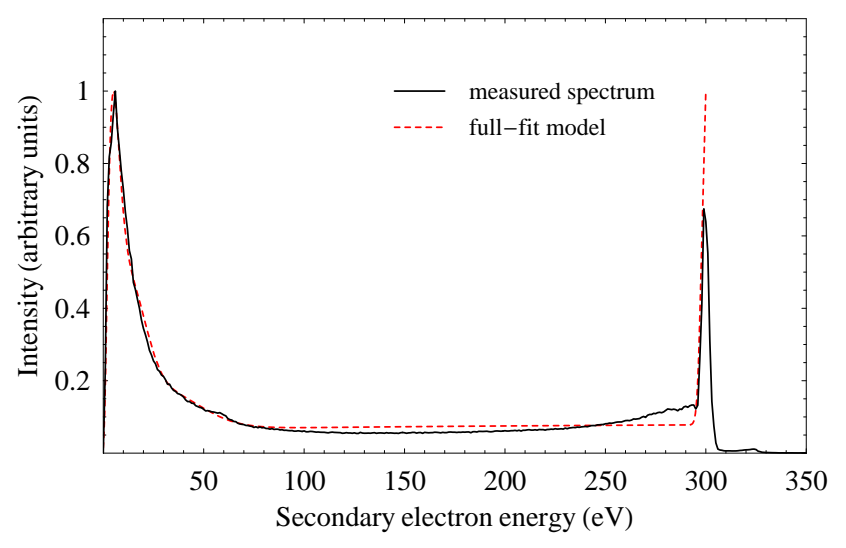

Figure 1: Secondary electron energy spectrum $d \delta / d E$ for $E_{0}=300 \mathrm{eV}$ (measured data and our model). The energy spectrum consists of the true secondary electrons (roughly the portion below $40 \mathrm{eV}$ ), the inelastic or re-diffused electrons (broad flat portion), and the elastically reflected electrons (peak at $E \simeq E_{0}$ ). The contributions to $\delta\left(E_{0}\right)$ are $44 \%, 48 \%$, and $8 \%$ respectively. The peak of the truesecondary component is at $\sim 5 \mathrm{eV}$ with a FWHM=12 eV.

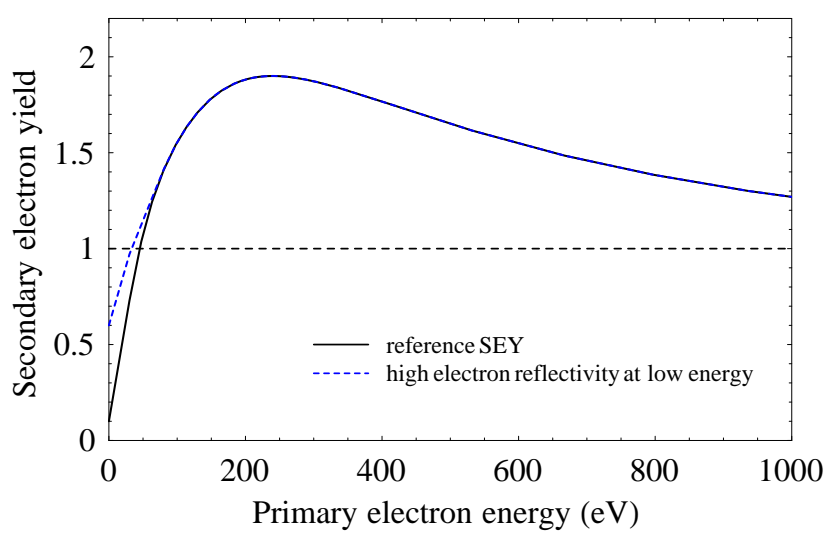

Figure 2: Our model SEY vs. primary electron energy, for $\delta_{\max }=1.9$ and $E_{\max }=240 \mathrm{eV}$. The case with $\delta(0)=0.6$ is also shown.

\subsection{Simulation Model}

In our simulation we assume the LHC proton beam to be composed of identical, evenly-spaced, proton bunches with population $N_{p}=1.05 \times 10^{11}$ separated by $\tau_{g}$. We assume that the bunch density is a $3 \mathrm{D}$ Gaussian distribution with rms sizes $\sigma_{x}, \sigma_{y}, \sigma_{z}$. We simulate the passage of the beam either in a dipole or in a field-free (FF) section in the arcs. We make the approximation that the vacuum chamber is made of a perfectly-conducting copper pipe of elliptical cross-section with semi-axes $(a, b)$. The electron generation by photons hitting the wall is represented by the product of two parameters, $Y^{\prime} \times N_{\gamma}$, where $Y^{\prime}$ is the effective photoelectric yield per penetrated photon, and $N_{\gamma}$ is the number of photons hitting the wall of the chamber whose energy is above $4 \mathrm{eV}$, per bunch passage. We assume that the time distribution of the generated photoelectrons is proportional to the instantaneous bunch intensity.

The scrubbing of the surface due to continued photon and/or electron bombardment leads to a conditioning effect [8-10] that is responsible for a decrease of $\delta_{\text {max }}$. We assume the initial value to be $\delta_{\max }=1.9$, while $\delta_{\max }=1.1$ represents the value after conditioning. Other electron emission parameters have been shown to be affected by the conditioning process. In our calculations we assume that both $Y^{\prime}$ and $E_{\max }$ decrease under the combined effect of particle and radiation bombardment [9] concomittantly with $\delta_{\max }$, as specified in Table 1 .

The photoelectrons are simulated by macroparticles. The secondary electron mechanism adds to these a variable number of macroparticles, generated stochastically according to the SEY model described above. The bunch is divided into slices, so that the macroparticles experience $N_{k}$ kicks during the bunch passage. We typically choose the full separation between the head kick and the tail kick to be $5 \sigma_{z}$. We also divide the empty buckets into $N_{g}$ intermediate steps. The space-charge force is calculated and applied at each slice during the bunch passage, and each step in the empty gap. The image forces of both protons and electrons are taken into account, assuming a perfectly conducting wall. In all results presented here, the proton beam is assumed to be a static distribution of given charge and shape moving on its nominal closed orbit, while the electrons are treated fully dynamically. Typical parameter values used in the simulations are shown in Table 1.

\section{RESULTS AND DISCUSSION}

The build-up of the electron cloud in a dipole section during the passage of the beam is shown in Fig. 3. The electrons gradually increase in number during successive bunch passages until, owing to the space-charge forces, a balance is reached between emitted and absorbed electrons. The estimated average number of electrons in a dipole vacuum chamber is $7 \times 10^{10}$ in the case of $\delta_{\max }=1.9$.

The power deposited by the electrons hitting the wall on the beam screen in an LHC arc is shown in Table 2, both for a field-free region (FF) and for a dipole magnet section.

The simulated heat load computed with the halfGaussian model is in very good agreement with the CERN results [4]. With the full-fit model, the estimated heat load increases by a factor $\sim 4$ relative to the half-Gaussian model. A comparison between the two models considered here is shown in Fig. 4. We conclude from this comparison that, in the case of LHC, the rediffused and elasticallyreflected electrons introduced with this model contribute 


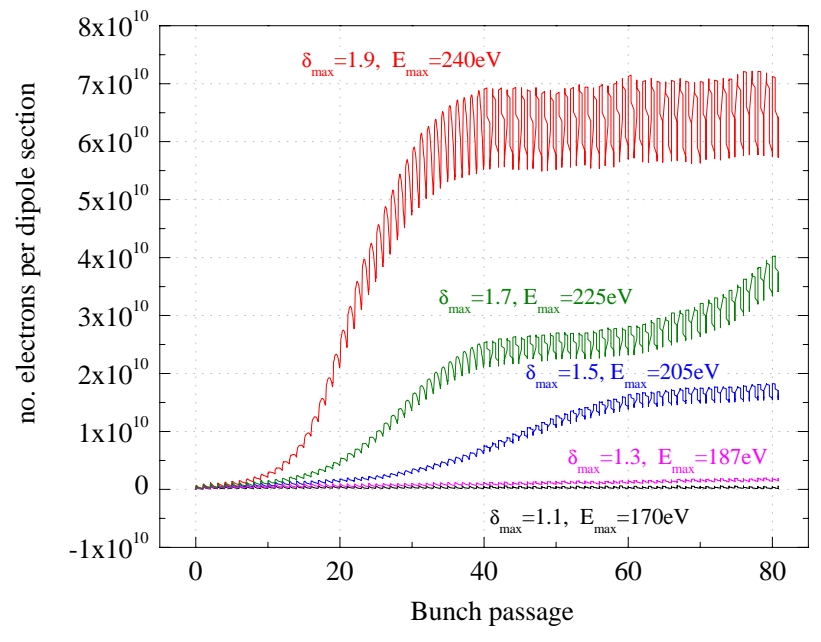

Figure 3: Build-up of the electron cloud: simulated number of electrons in a dipole during the passage of an 81-bunch batch, at nominal beam intensity (full-fit model).

Table 2: Simulated power deposition (units: W/m).

\begin{tabular}{lccrr}
\hline \hline Model & $\delta(0)$ & $\delta_{\max }$ & FF & Dipole \\
\hline half-Gaussian & 0.1 & 1.9 & 6.44 & 1.81 \\
half-Gaussian & 0.1 & 1.1 & 0.92 & 0.035 \\
\hline full fit & 0.1 & 1.9 & 14.4 & 8.69 \\
full fit & 0.1 & 1.1 & 0.57 & 0.02 \\
\hline full fit & 0.6 & 1.9 & 49.5 & 16.3 \\
full fit & 0.6 & 1.1 & 1.2 & 0.06 \\
\hline \hline
\end{tabular}

significantly to the heat load.

For the case of $\delta(0)=0.6$, which is a rather high value for the SEY at low energy, the simulated heat load is significantly larger than for $\delta(0)=0.1$, as seen in Table 2. If we truncate the full-fit model by leaving out the rediffused and elastically reflected electrons and retaining only the truesecondary component of the spectrum, the heat load in a dipole is $3.2 \mathrm{~W} / \mathrm{m}$ for $\delta(0)=0.1$ and $\delta_{\max }=1.9$, as opposed to $8.69 \mathrm{~W} / \mathrm{m}$ for the full model. These results indicate a strong sensitivity to details of the SEY at low energy that calls for further experimental and theoretical investigations.

\section{ACKNOWLEDGMENTS}

We are grateful to I. Collins and F. Zimmermann for many discussions. We are indebted to R. Kirby for providing us data on the SEY. We are grateful to NERSC for supercomputer support.

\section{REFERENCES}

[1] For a recent review, see G. Rumolo, F. Ruggiero and F. Zimmermann, PRST-AB 4, 012801 (2001) (erratum: 4, 029901 (2001)); see also F. Zimmermann, these proceedings.

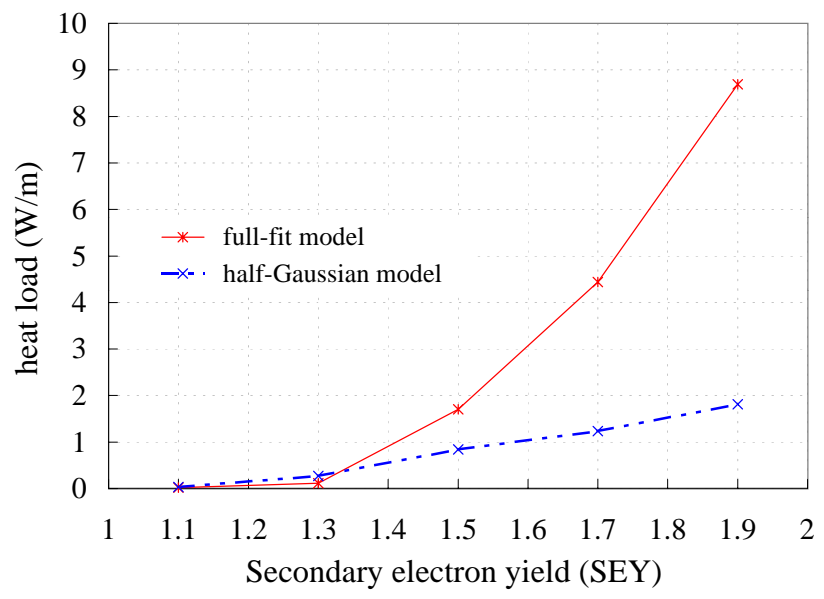

Figure 4: Simulated power deposition on the beam screen of a dipole magnet. Results obtained with the full-fit model (including true-secondary, rediffused and elastically reflected electrons), compared with the half-gaussian model.

[2] See the LHC ECE website, http://wwwslap.cern.ch/ collective/electron-cloud/electron-cloud.html

[3] M. A. Furman, LBNL-41482/CBP Note 247/LHC Project Report 180, May 20, 1998. http://www.lbl.gov/ miguel/

[4] F. Zimmermann, Proc. XI Chamonix Workshop, Jan. 2001, http://cern.web.cern.ch/CERN/Divisions/SL/publications/ chamx2001/contents.html

[5] H. Seiler, J. Appl. Phys. 54(11), Nov. 1983, pp. R1-R18.

[6] M. A. Furman and G. R. Lambertson, Proc. Intl. Workshop on Multibunch Instabilities in Future Electron and Positron Accelerators (MBI-97), KEK, Tsukuba, Japan, 1518 July 1997 (KEK Proceedings 97-17, Dec. 1997, p. 170); http://www.lbl.gov/ miguel/MBI97-ECI-PEPII.pdf

[7] R. Kirby, private communication.

[8] O. Brüning et. al, Proc. PAC99 (NY City), p. 2629.

[9] V. Baglin, B. Henrist and N. Hilleret, http://wwwslap. cern.ch/collective/electron-cloud/baglin/EPA_sey-14-042000.pdf, esp. p. 11-14.

[10] R. E. Kirby and F. K. King, SLAC-PUB-8212, Oct. 2000, to be published in NIMPR. 